\title{
In-situ infrared thermography measurements to master transmission laser welding process parameters of PEKK
}

\author{
M. Villar ${ }^{\mathrm{a}}$, C. Garnier ${ }^{\mathrm{a}, *}$, F. Chabert ${ }^{\mathrm{a}}$, V. Nassiet ${ }^{\mathrm{a}}$, D. Samélor ${ }^{\mathrm{b}}$, J.C. Diez ${ }^{\mathrm{c}}$, A. Sotelo ${ }^{\mathrm{c}}$, \\ M.A. Madre ${ }^{\mathrm{c}}$ \\ a LGP-ENIT-INPT, Université de Toulouse, 47 Avenue d'Azereix, Tarbes cedex BP1629-65016, France \\ ${ }^{\mathrm{b}}$ CIRIMAT, Université de Toulouse, CNRS, INPT, UPS, ENSIACET-4 allée Emile Monso, Toulouse cedex 4 BP44362-31030, France \\ ${ }^{c}$ Institute of materials science of Aragon, CSIC-University of Zaragoza, Calle de Pedro Cerbuna, 12, Zaragoza 50009, Spain
}

A R T I C L E I N F O

\section{Keywords:}

Assembling process

Transmission laser welding

Polymeric material

PEKK

Infrared thermography

Variability test

\begin{abstract}
A B S T R A C T
The temperature field along the thickness of the specimens has been measured during transmission laser welding. Polyetherketoneketone (PEKK) is a very high performance thermoplastic with tunable properties. We have shown that this grade of PEKK can be turned to quasi-amorphous or semi-crystalline material, due to its slow kinetics of crystallization. Its glass transition temperature is $150{ }^{\circ} \mathrm{C}$. The effect of its crystalline rate directly impacts its optical properties: the transmittance of quasi-amorphous PEKK is about $60 \%$ in the NIR region (wavelength range from 0.4 to $1.2 \mu \mathrm{m}$ ) whereas it is less than $3 \%$ for the semi-crystalline material. The welding tests have been carried out with an $808 \mathrm{~nm}$ laser diode apparatus. The heat field is recorded during the welding experiment by infrared thermography with the camera sensor perpendicular to the lasersheet and to the sample's length to focus on the welded interface. The study is divided in two steps: firstly, a single specimen is irradiated with an energy density of $22 \mathrm{~J} . \mathrm{mm}^{-2}$ : the whole sample thickness is heated up, the maximum temperature reaches $222 \pm 7{ }^{\circ} \mathrm{C}$. This temperature corresponds to about $\mathrm{T}_{\mathrm{g}}+70^{\circ} \mathrm{C}$, but the polymer does not reach its melting temperature. After that, welding tests were performed: a transparent (quasi-amorphous) sample as the upper part and an opaque (semi-crystalline) one as the lower part were assembled in static conditions. The maximum temperature reached at the welded interface is about $295{ }^{\circ} \mathrm{C}$ when the upper specimen is irradiated for $16 \mathrm{~s}$ with an energy density of $28 \mathrm{~J} . \mathrm{mm}^{-2}$. The temperature at the welded interface stays above $\mathrm{T}_{\mathrm{g}}$ during $55 \mathrm{~s}$ and reached the melting temperature during $5 \mathrm{~s}$ before rapid cooling. These parameters are suitable to assemble both polymeric parts in a strong weld. This work shows that infrared thermography is an appropriate technique to improve the reliability of laser welding process of high performance thermoplastics.
\end{abstract}

\section{Introduction}

High performance thermoplastics gradually replace metallic alloys in some industrial fields, such as aerospace and power electronics. Indeed, they attest high mechanical strength combined to heat resistance higher than $200^{\circ} \mathrm{C}$ as well as resistance to corrosion, with the advantage of lightness. The latter results in progress towards sustainability. Among them, the polyaryletherketone (PAEK) family has been demonstrated to be the most resistant to thermo-oxidative degradation [1]. In particular, polyetherketoneketone (PEKK) with an Ether/Ketone ratio of $\frac{1}{2}$, keeps a conservative modulus higher than $1 \mathrm{GPa}$ up to its glass transition $\left(\mathrm{T}_{\mathrm{g}}\right)$ at about $150{ }^{\circ} \mathrm{C}$. The advantage of PEKK is its slower crystallization, allowing to easily turn PEKK into amorphous or semi-crystalline polymer, compared to well-known polyetheretherketone (PEEK). More knowledge and know-how about assembling processes of PAEK would seemingly lead to widespread industrial uses.

Among assembling processes for thermoplastics, laser welding is an environmentally-friendly, fast, reliable and non-contact process [2]. Welding by transmission requires the upper part to be transparent to the laser wavelength whereas the inferior part absorbs the same wavelength. Thus, the energy of the beam is stopped at the interface, allowing both polymeric parts to heat up. Early theories on welding strength were based on diffusion [2]. Upon temperature, the interdiffusion of polymeric chains takes place at the interface, creating macromolecular entanglements between both parts, resulting in a strong assembly. Laser welding is in principle applicable to any thermoplastic, provided that the upper part is transparent to the laser wavelength. This process is already applied in the industry with common polymers such as polycarbonate and PMMA [3].

\footnotetext{
* Corresponding author.

E-mail address: c.garnier@enit.fr (C. Garnier).
} 
Since amorphous polymers are commonly transparent to laser beam, laser transmission welding is feasible. Thus, the beam goes through the material without inducing major changes in its chemical structure. From a physical point of view, a theory to describe the adhesion of similar polymers, also called autohesion, based on the diffusion of macromolecules above $T_{g}$ was proposed in 1960s by Voyutskii [4] and Vanenin [5]. Then, the theory of reptation exposed by De Gennes [6,7] described the motion of polymeric chains and was successful in predicting the molecular weight dependency of the self-diffusion. From these theories, a number of attempts have been made in order to link the process parameters to the interfacial strength in welding processes. Among them, Wool's model [8], predicting the mechanical energy required to separate two welded polymeric parts as a function of time, pressure, temperature and molecular weight, appears to best fit the experimental data.

Welding a semi-crystalline polymer is a challenge: most of the theories predicting the interfacial strength do not take into account the role of crystallinity on self-diffusion and the role of the kinetics of crystallization. Moreover, laser transmission welding requires the upper part to be transparent and to stay transparent to the laser beam all along the process. Semi-crystalline polymers such as PAEK are opaque to visiblenear infrared light. Nevertheless, some studies go inside more knowledge about transmission laser welding of PEEK: Amanat et al. [9] give the parameters ranges to get strong welds of $250 \mu \mathrm{m}$ thick films with an infrared absorbing medium at the interface, with a pulsed fiber laser with a wavelength of $1060 \mathrm{~nm}$. As a result, some bubbles are trapped at the interface: the explanation given by the authors is the presence of water in the specimens. Potente et al. [10] welded undried and pre-dried PEEK, they noticed bubbles in the undried PEEK welds only. Sometimes, heat damages are reported [9] on amorphous PEEK at a power of $20 \mathrm{~W}$ with a focal plane speed of $4 \mathrm{~mm} \cdot \mathrm{s}^{-1}$. Despite the fact that authors agree about the effect of water, the process parameters, such as power and sample speed, are not well defined yet. Additionally, the experimental studies of laser welding process lack conclusive links between process parameters and their effect on assemblies. For instance, the effect of laser power on the temperature of specimens is indefinite until now.

Measuring the temperatures involved during the laser welding process is meaningful for many reasons: (a) checking that the temperature reached is high enough to insure self-diffusion according to autohesion theories to get interfacial strength (b) checking that the temperature inside the polymeric parts stays low enough to avoid thermal degradation of the material, and (c) locating the Heat-Affected Zone (HAZ), the latter has possibly undergone morphological changes and thus, modification of properties and dimensions. In-situ temperature measurements would allow getting insight the transmission laser welding process to make it more reliable.

Measuring the temperature at the interface is a demanding task because both polymeric parts are in closed contact all along the process. So, contact-free temperature measurement techniques such as infrared (IR) thermography have been implemented to the laser welding process in some studies [11-14]. In parallel to the experimental work, these authors have developed a numerical simulation to predict the temperature at the interface. To validate their model, the chosen materials are either PMMA as semi-transparent element and ABS/PC as absorbent part or, in another study, polycarbonate based FRP (fiber reinforced polymer). Both are amorphous polymers, with well-known transmission, reflection and absorption factors at near- and medium-IR spectrum. To date, the laser welding of PEKK has not been reported, due to the novelty of this high-performance thermoplastic. Also, the originality of this work is to weld two samples of the same polymer tuned into amorphous (A-PEKK, transparent) and semi-crystalline (C-PEKK, opaque) state.

Although IR thermography seems to be suited to the transmission laser welding process, no study uses it as a technique for controlling temperatures distribution along the sample's thickness. In the existing studies, measurements were performed with the camera sensor parallel to the welded interface. With such an experimental setup, the temperature is measured at the upper or lower surface of the polymeric parts. These

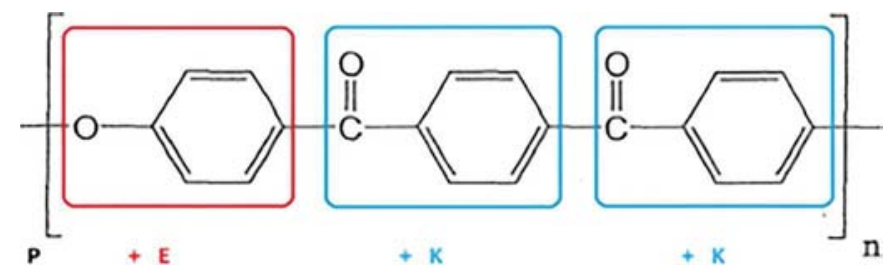

Fig. 1. Chemical structure of PEKK.

calculated/experimental results were implemented to numerical simulation, using a hybrid code that combines Mie theory $[15,16]$ and the Monte Carlo method [17] to quantify the scattering phenomena. The experimental and numerical results match for the temperatures measured at the upper and lower surfaces, nevertheless, the temperature at the interface is forecast from these measurements but not really measured. With the camera sensor perpendicular to the lasersheet and perpendicular to the sample's length, the temperature at the interface of welded assembly is reported for the first time.

In this work, the feasibility of determining the temperature distribution at the interface along the sample's thickness during the welding process of PEKK is outlined. To achieve this, the heat field is recorded by infrared thermography with the camera sensor perpendicular to the lasersheet and perpendicular the welded interface. This study is divided in two steps: Firstly, a test of laser beam/specimen interaction was performed in order to bring a better understanding of fast heating effects due to high concentrated power laser spot on a semi-crystalline PEKK. Then, for the welding test, an assembly made of amorphous PEKK as the upper part and semi-crystalline PEKK as the lower part was used. The effects of process parameters on the properties of PEKK are explained.

\section{Materials and methods}

A high performance PAEK, the polyetherketoneketone (PEKK) Kepstan 6002 by Arkema was chosen. Its chemical structure is shown in Fig. 1, it is made of ether and ketone copolymers in $\frac{1}{2}$ ratio.

Quasi-amorphous and semi-crystalline PEKK specimens were used for the welding test. In the following, quasi-amorphous PEKK will be referred as A-PEKK and semi-crystalline PEKK as C-PEKK. Both specimens were processed by compression molding from dried pellets. An $800 \mathrm{kN}$ Pinette Emidecau LAB 800P hot plates press was used; with controlled heating and cooling temperatures from 2 to $10^{\circ} \mathrm{C} \cdot \mathrm{min}^{-1}$. For controlling the crystalline rate and plate dimensions, the samples were made in two steps. In the first step, the pellets were melted at $340^{\circ} \mathrm{C}$, slightly compressed and cooled down to room temperature. The cooling rate was $2{ }^{\circ} \mathrm{C} . \mathrm{min}^{-1}$ for the crystallized sample; and liquid nitrogen was used for a fast cooling rate to get the quasi-amorphous sample. In the second step, the samples were flattened up to $2 \mathrm{~mm}$ thick while heating at $2{ }^{\circ} \mathrm{C} \cdot \mathrm{min}^{-1}$ until glass transition in order to remove any residual stress. The compression molded specimens were dried during $24 \mathrm{~h}$ at $140{ }^{\circ} \mathrm{C}$ to remove water before welding.

Transmission factors and reflection factors in the wavelength range of 400-1100 nm have been measured with a Perkin Elmer Lambda 19 spectrophotometer equipped with an integrating sphere. The incidence beam angle was $8^{\circ}$ with respect to the perpendicular direction of the sample surface. Before the experiments, the device was calibrated with a BaS background. Tests were performed at $20^{\circ} \mathrm{C}$.

Transmission factors in the wavelength range of 3-5 $\mu \mathrm{m}$ have been measured with a Perkin Elmer Spectrum One spectrophotometer in the transmission mode. The incidence beam angle is $0^{\circ}$ with respect to the perpendicular direction of the sample surface. Tests were performed at $20^{\circ} \mathrm{C}$.

Differential Scanning Calorimetry (DSC) scans were carried out with a DSC Q200 from TA Instruments calibrated with indium, starting at room temperature to $400{ }^{\circ} \mathrm{C}$ with a ramp of $5{ }^{\circ} \mathrm{C} \cdot \mathrm{min}^{-1}$. Samples were 

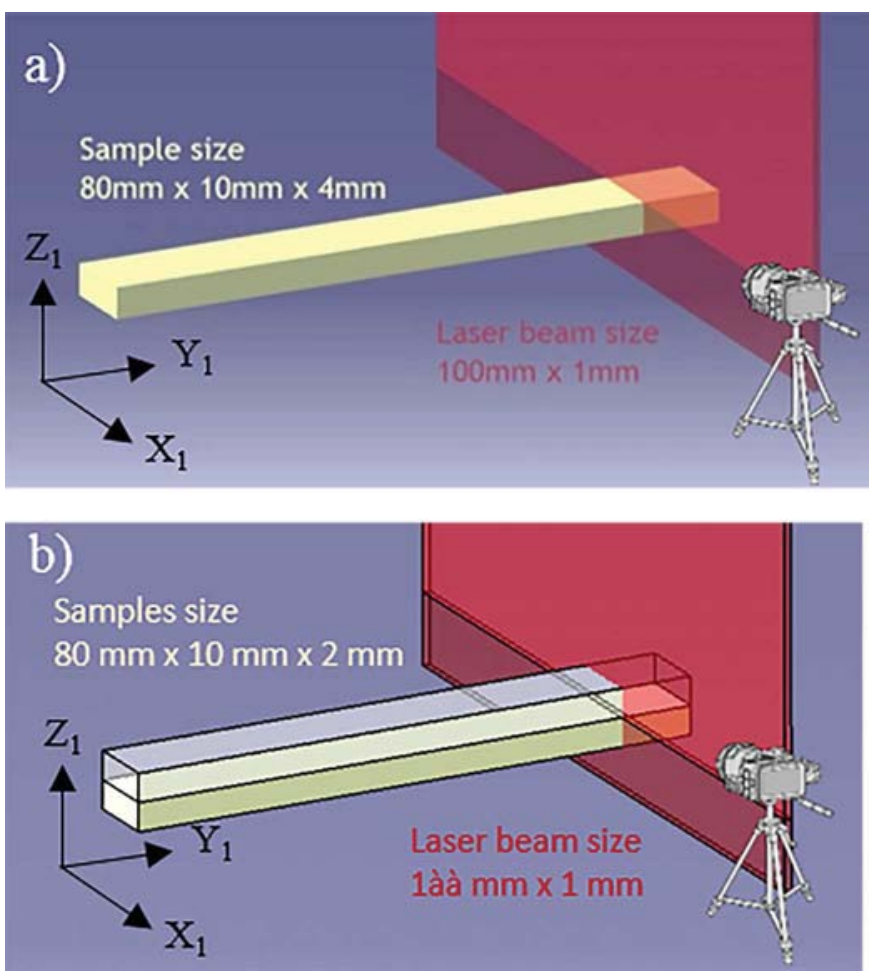

Fig. 2. Scheme of the projected laser beam on the specimen, a) single specimen, b) assembly.

studied as processed, by keeping their thermomechanical history of compression molding process, to be as close as possible to industrial conditions. Glass transition temperature $\left(\mathrm{T}_{\mathrm{g}}\right)$, cold crystallization temperature $\left(\mathrm{T}_{\mathrm{cc}}\right)$ and melting temperature $\left(\mathrm{T}_{\mathrm{m}}\right)$ were obtained. Cooling ramps were performed at $2{ }^{\circ} \mathrm{C} \cdot \mathrm{min}^{-1}$ and $10{ }^{\circ} \mathrm{C} \cdot \mathrm{min}^{-1}$ from 400 to $20^{\circ} \mathrm{C}$. Crystallization enthalpy and melting enthalpy were calculated by integration of the area of the exothermic and endothermic peaks respectively.

Experimental near infrared laser device performs a continuous $808 \mathrm{~nm}$ beam, projecting a single $100 \times 1 \mathrm{~mm}^{2}$ lasersheet, as represented in Fig. 2. Laser source is fed by 90 diodes branched on 6 stacks of 15 diodes. Laser power is set by diode feeding intensity, starting power emission at $13 \mathrm{~A}$ and reaching $230 \mathrm{~W}$ for $40 \mathrm{~A}$. Nonetheless, power is spread out all along the lasersheet, so only actual irradiated zone must be considered in interaction for energy calculations. Even though laser welding is usually performed in dynamic setup, static tests were carried out for a more accurate interfacial measurement, and to suppress parasite accumulative thermal effects appearing for dynamic laser welding.

Infrared temperature fields measurements were performed with an

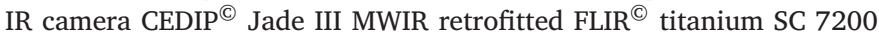
with a thermal resolution of $20 \mathrm{mK}$ at $30^{\circ} \mathrm{C}$. The used detector is a midwave Indium Antimonide (InSb) one and the sensor is a close-up x1 covering a spectral range from 3.70 to $5.15 \mu \mathrm{m}$ with a focal plane array (FPA) windowing of $7.6 \times 9.9 \mathrm{~mm}^{2}$. Based on literature, we assume the reflectivity of the materials is much less than 1 [11] and so, the reflection factor is assumed to be less than $15 \%$ for amorphous specimens. The detector resolution is $320 \times 256$ pixels and the pitch of the sensor is $30 \mu \mathrm{m}$. So on, the pixel size on both directions is $0.03 \mathrm{~mm}$. Calibration and data recording were managed by Altair software.

Specimens were laid out $300 \mathrm{~mm}$ far from the close up $\mathrm{x} 1$ sensor to have the best sharpness. In order to measure the interfacial temperature and the distribution of thermal field along both directions (thickness and length of the sample), side-by recordings were performed scoping the region of interest (Fig. 3). The whole experiments were done in a $25^{\circ} \mathrm{C}$ in a thermoregulated dark room to reduce the effect of environment on IR measurement. With regard to measure the rapid temperature rise
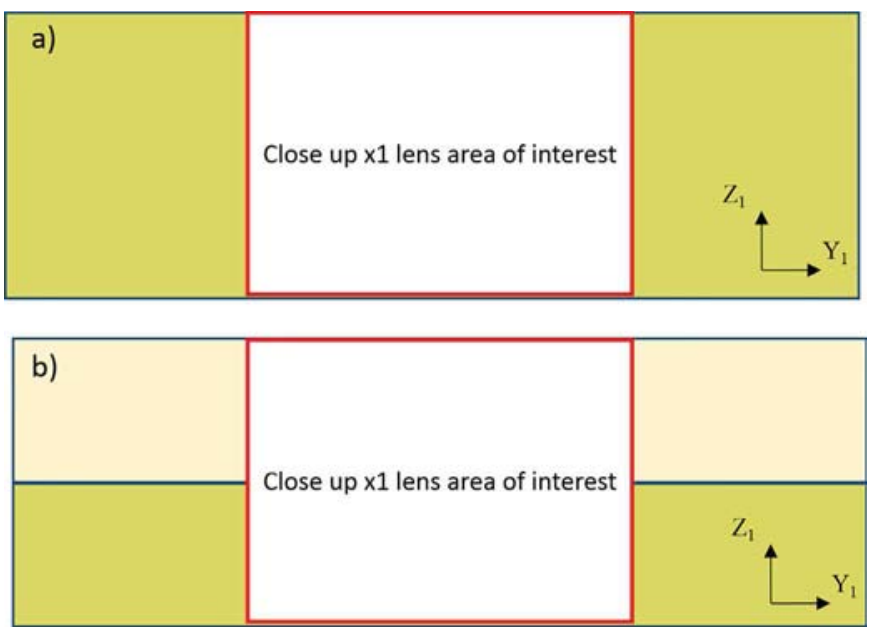

Fig. 3. Infrared area of interest of the samples, a) single specimen, b) assembly.

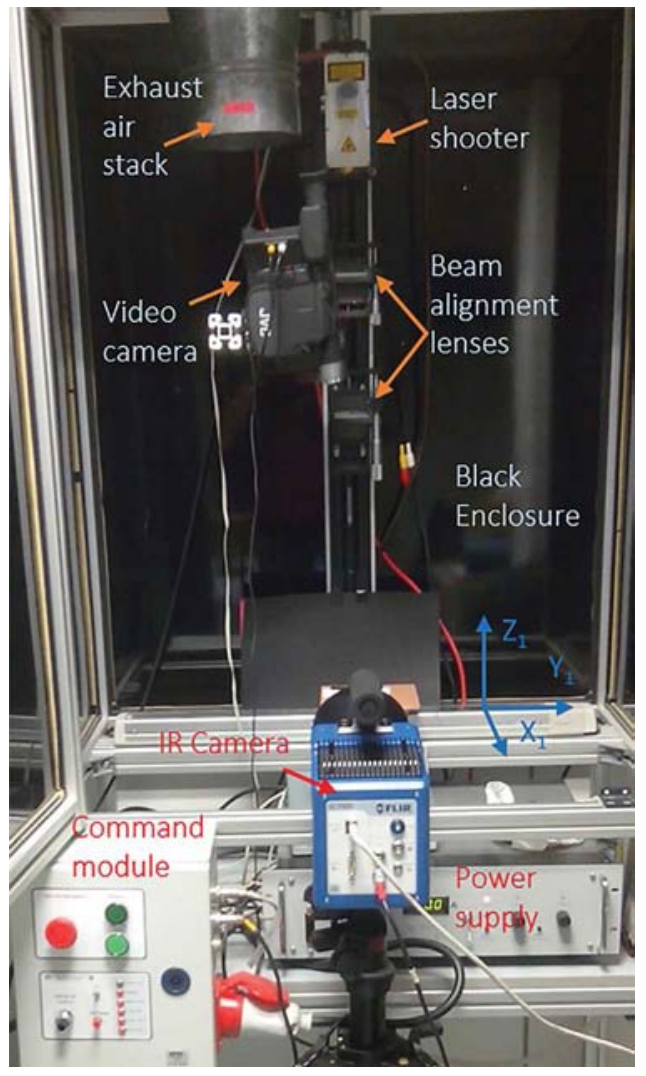

Fig. 4. Experimental laser device.

during laser irradiation, integration time was set up at $16 \mu$ s, obtaining a temperature range from 140 to $300{ }^{\circ} \mathrm{C}$. IR Movies were recorded at $125 \mathrm{~Hz}$ with 5 images averaging conducive to minimizing the random noise. So on, the effective frequency was $25 \mathrm{~Hz}$. The whole experimental set up is shown in Figs. 4 and 5.

\section{Results and discussion}

Unless the technique of DSC is controverted for the analysis of the crystallinity of PAEK because of the nearness of melting and crystallization [18], we choose it to compare the thermal transitions of the quasi-amorphous and crystallized materials because of the simplicity of use. The thermal transitions at the first heating of A-PEKK and C- 


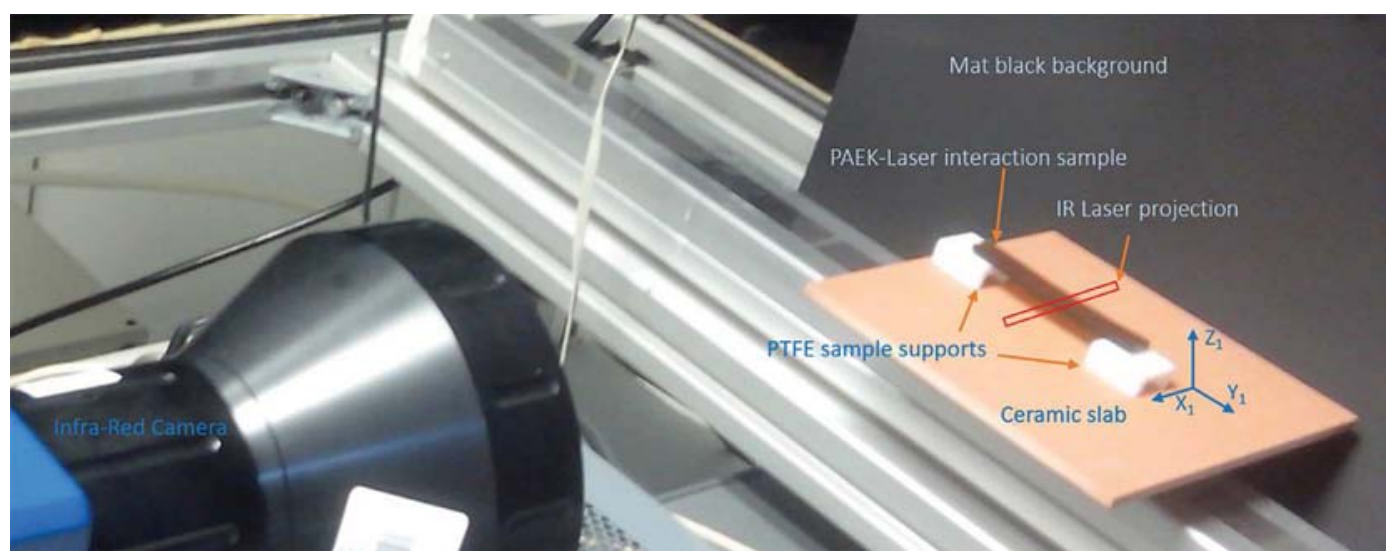

Fig. 5. Layout of infrared recording scene.

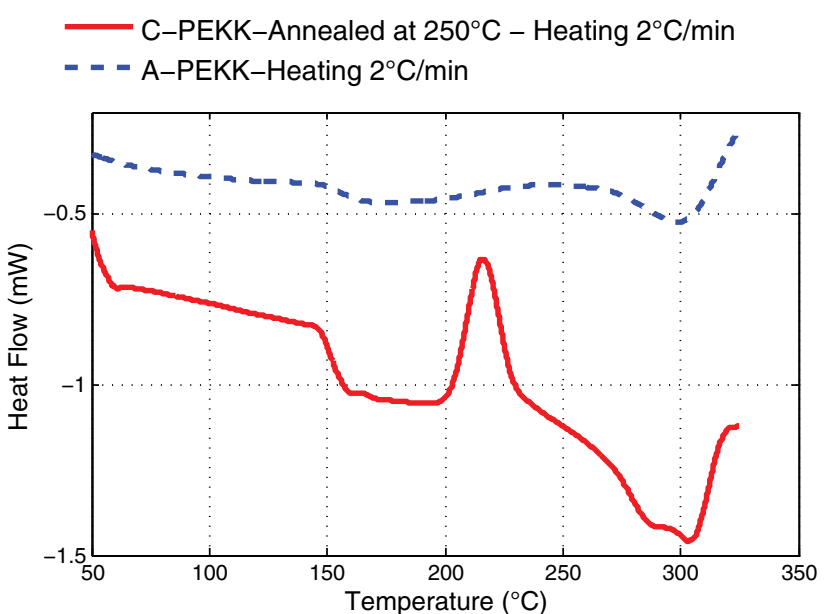

Fig. 6. DSC thermogram of A-PEKK and C-PEKK: first heating at $2^{\circ} \mathrm{C} \cdot \mathrm{min}^{-1}$ of molded samples.

PEKK are seen in Fig. 6. For both materials, the thermograms display a glass transition at $150{ }^{\circ} \mathrm{C}$, when the macromolecular chains have gained enough mobility to move slightly. Then, for A-PEKK, a cold crystallization peak is observed with a maximum at $220^{\circ} \mathrm{C}$ followed by a double melting peak centered at $300^{\circ} \mathrm{C}$. The enthalpy of crystallization $\left(\Delta \mathrm{H}_{\mathrm{c}}=18.6 \pm 0.5 \mathrm{~J} \mathrm{~g}^{-1}\right)$ is lower than the enthalpy of melting $\left(\Delta \mathrm{H}_{\mathrm{mA}}=20.0 \pm 0.5 \mathrm{~J}^{-1} \mathrm{~g}^{-1}\right)$ indicating that the specimens were slightly crystalline before testing, due to the compression molding process. The crystalline rate is usually given by: $\Delta \mathrm{H}_{\mathrm{m}} / \Delta \mathrm{H}_{100 \%}$, assuming $\Delta \mathrm{H}_{100 \%}$ is $122 \mathrm{J.g}^{-1}$ like for PEEK [19], it gives an insignificant rate of $0.01 \%$ for amorphous A-PEKK. For C-PEKK, no cold-crystallization occurs, the cooling rate applied during the compression process is slow enough to allow the macromolecules to self-organized into crystalline phase at most as they can. The enthalpy of melting is $\Delta \mathrm{H}_{\mathrm{mC}}=25.6 \pm 0.5 \mathrm{J.g}^{-1}$, corresponding to $20.9 \%$ of crystallinity still assuming $\Delta \mathrm{H}_{100 \%}=122 \mathrm{~J}_{\mathrm{g}} \mathrm{g}^{-1}$. Since the C-PEKK is processed with a long annealing time, this melting enthalpy approaches the maximum crystallinity reached by this grade of PEKK.

Welding a semi-crystalline polymer is a challenge, since the upper part as to stay transparent to the laser beam all along the process. As soon as the crystalline rate increases, any semi-crystalline polymer turns transparent to semi-transparent to opaque for highest crystalline rates. It is well known that it is possible to control the crystallinity by applying a cooling rate faster than the kinetics of crystallization. This way, PEKK can be obtained as transparent or opaque material. The kinetics of crystallization is slow for this grade of PEKK compared to other PAEK

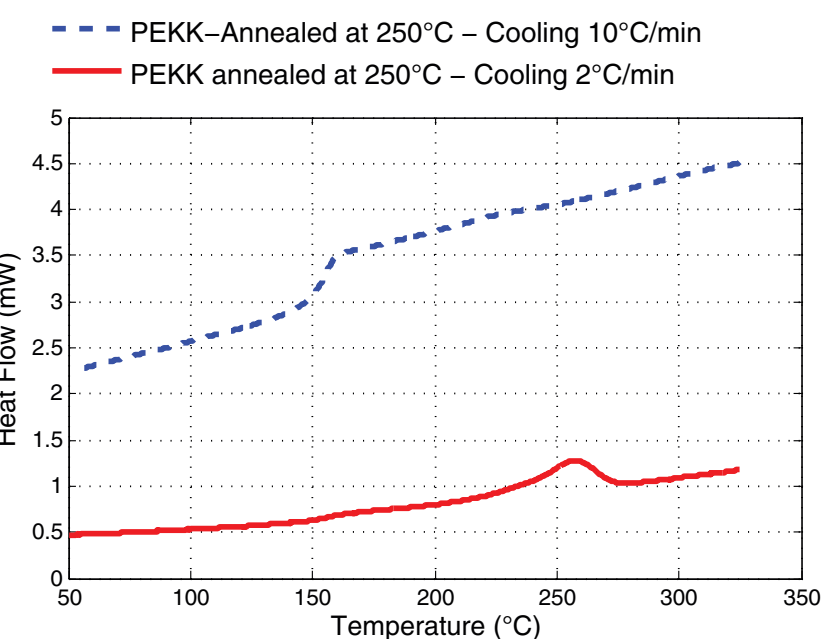

Fig. 7. DSC thermogram of PEKK: cooling ramps from melting state at $380{ }^{\circ} \mathrm{C}$.

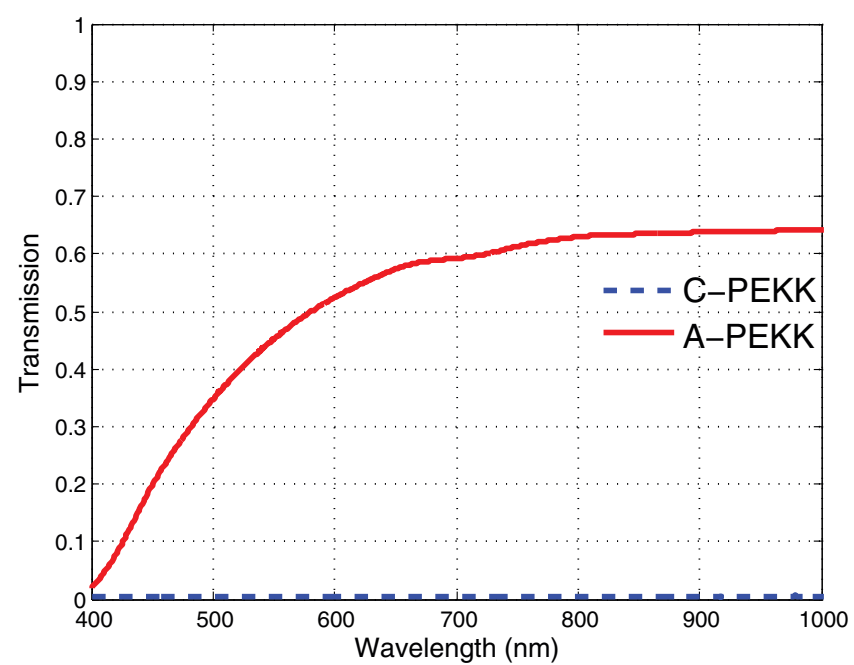

Fig. 8. Transmission factor for A-PEKK and C-PEKK measured by spectrophotometry.

[16], allowing thoroughly controlling the crystallinity along the processing steps. To go further the kinetics of crystallization of this grade of PEKK, cooling ramps at various rates have been performed, as seen in Fig. 7. From the melting state, no crystallization occurs when the cooling rate is $10^{\circ} \mathrm{C} \cdot \mathrm{min}^{-1}$ or faster. The glass transition is broad at $150{ }^{\circ} \mathrm{C}$. At 


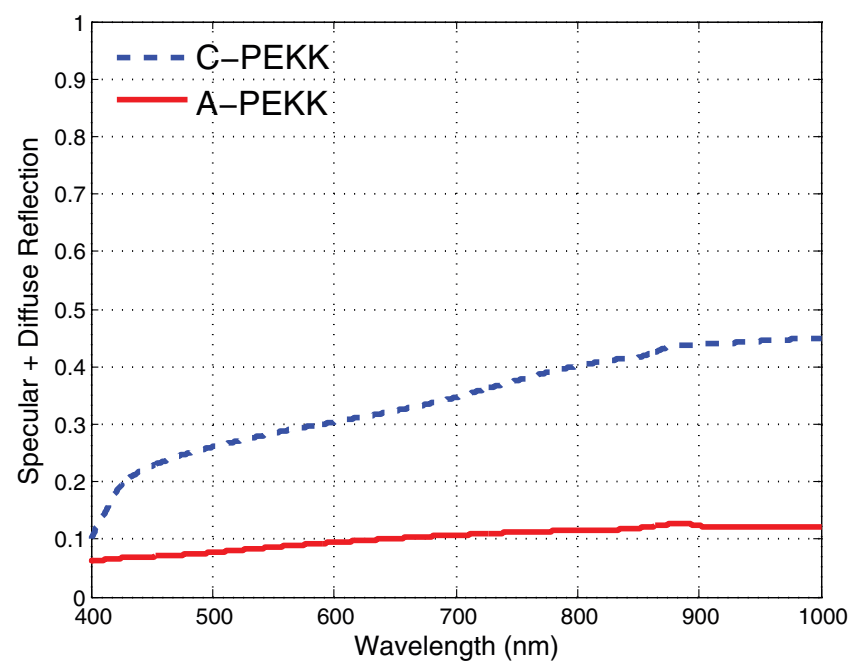

Fig. 9. Reflection factor for A-PEKK and C-PEKK measured by spectrophotometry with an integrating sphere.

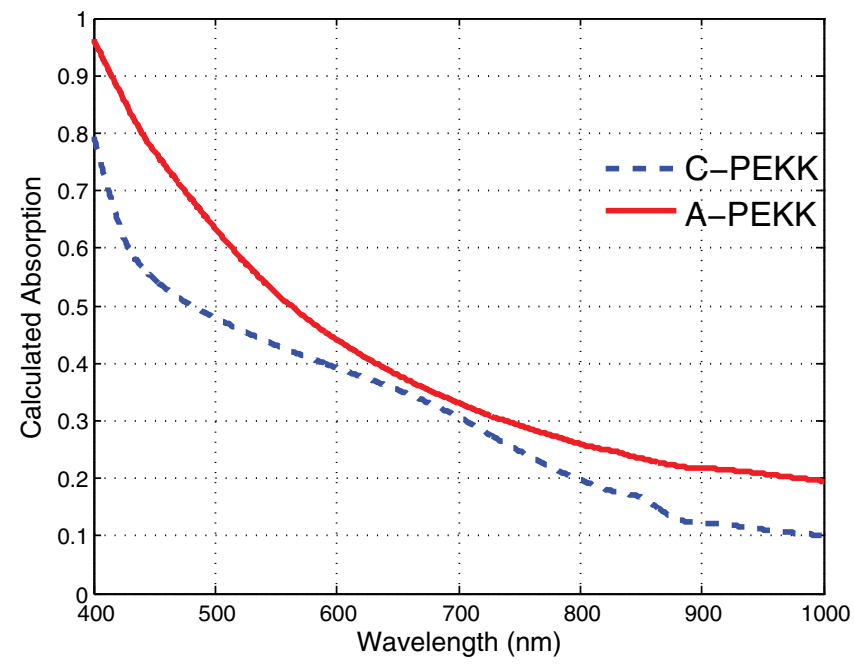

Fig. 10. Absorbance factor $(\alpha)$ for A-PEKK and C-PEKK, calculated with Eq. (1).

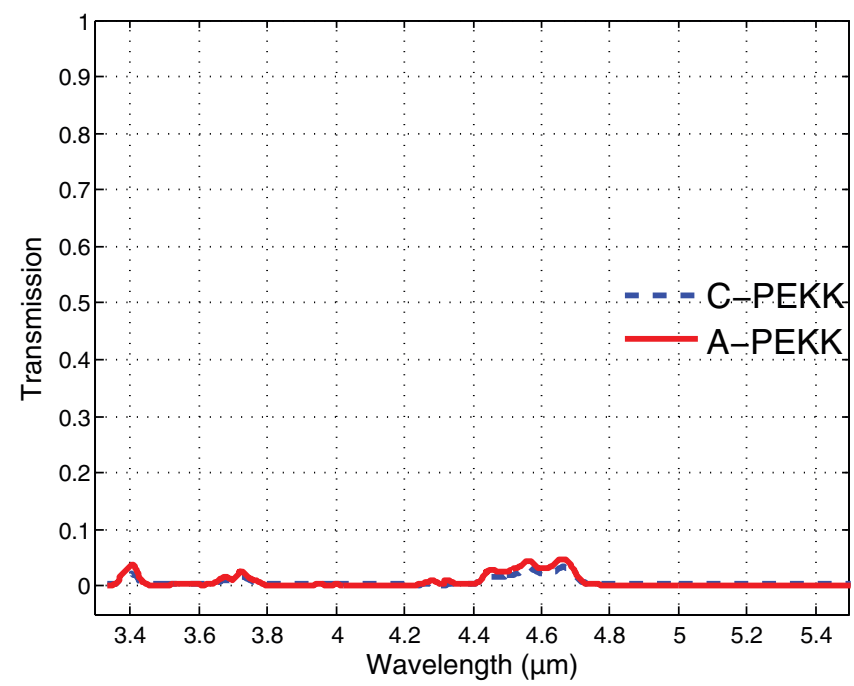

Fig. 11. Transmission factor for A-PEKK and C-PEKK measured by spectrophotometry.

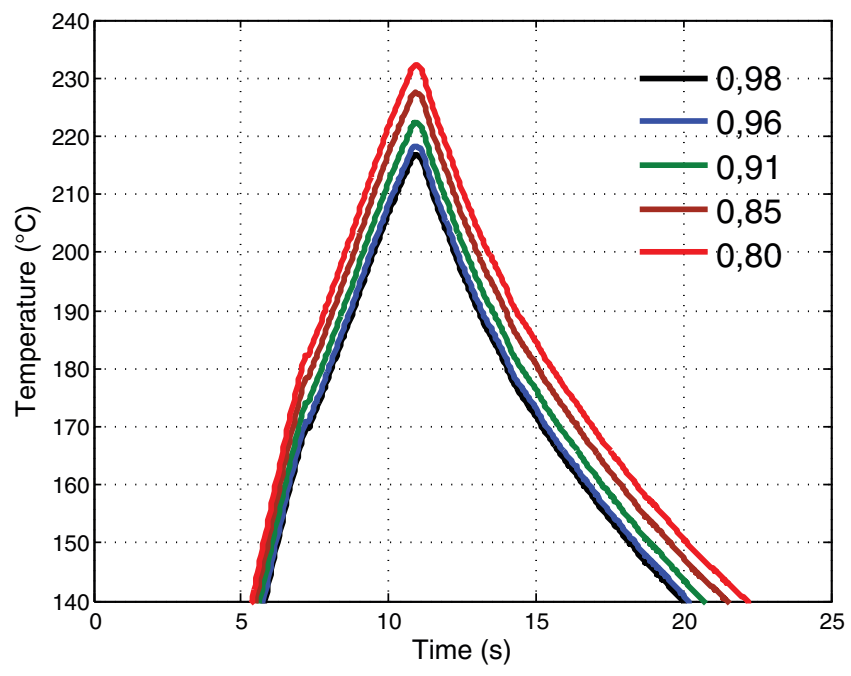

Fig. 12. Impact of the emissivity rate variability on maximum temperature.

Table 1

Impact of the emissivity variability on temperature measurements.

\begin{tabular}{llllll} 
Emissivity $\varepsilon$ & 0.80 & 0.85 & 0.91 & 0.96 & 0.98 \\
Max temperature $\left({ }^{\circ} \mathrm{C}\right)$ & 232.25 & 227.55 & 222.35 & 218.34 & 216.81 \\
$\Delta \mathrm{T}\left({ }^{\circ} \mathrm{C}\right)$ & +9.9 & +5.2 & - & -4.01 & -5.54 \\
\hline
\end{tabular}

$2{ }^{\circ} \mathrm{C} \cdot \mathrm{min}^{-1}$, an exothermic peak indicates the crystallization: it could be interpreted as the organization of the shortest chains, the latter having more mobility than the longest ones. The glass transition, still at $150{ }^{\circ} \mathrm{C}$ is less obvious when the cooling rate is lower. As a comparison, the crystallinity of PEEK is maximum even with a cooling rate of $25^{\circ} \mathrm{C} . \mathrm{min}^{-1}$ [16].

For the transmission laser welding process, the polymeric material used as top element has to be transparent enough for the laser beam to go through. In the same idea, the lower element of the assembly has to be absorbent to the wavelength. The optical properties of both A-PEKK and C-PEKK have been determined in the visible-near infrared range, from 400 to $1100 \mathrm{~nm}$. Any thermoplastic is transparent when amorphous and it turns to opaque when it is semi-crystalline.

The absorbance $A_{\lambda}$, transmittance $T_{\lambda}$ and reflectance $R_{\lambda}$ are linked to the intensity of the light flux $\varphi_{\lambda}^{\gamma}$ by Eq. (1), where ?? is $t$ for transmitted flux, $i$ for incident flux and $r$ for reflected flux:

$$
\begin{aligned}
& A_{\lambda}+T_{\lambda}+R_{\lambda}=1 \\
& T_{\lambda}=\frac{\varphi_{\lambda}^{t}}{\varphi_{\lambda}^{i}} \\
& R_{\lambda}=\frac{\varphi_{\lambda}^{r}}{\varphi_{\lambda}^{i}}
\end{aligned}
$$

The transmissivity coefficient and the reflectivity coefficient have been measured using a spectrophotometer with an integrating sphere. For the same temperature, the transmission factor is influenced by the surface roughness, the light incidence angle and the sample thickness. The results of transmission and reflection are shown in Figs. 8 and 9 respectively. The transmission of the amorphous PEKK is negligible between 200 and $400 \mathrm{~nm}$, then, the transmission factor increases to reach $65 \pm 5 \%$ at maximum for $1100 \mathrm{~nm}$. At the wavelength of interest $(808 \mathrm{~nm})$, the transmission factor is $60 \pm 5 \%$ at $20{ }^{\circ} \mathrm{C}$ for the $2 \mathrm{~mm}$ thick samples. For the semi-crystalline sample, the transmission is about $3 \%$ all over the studied wavelength range. 


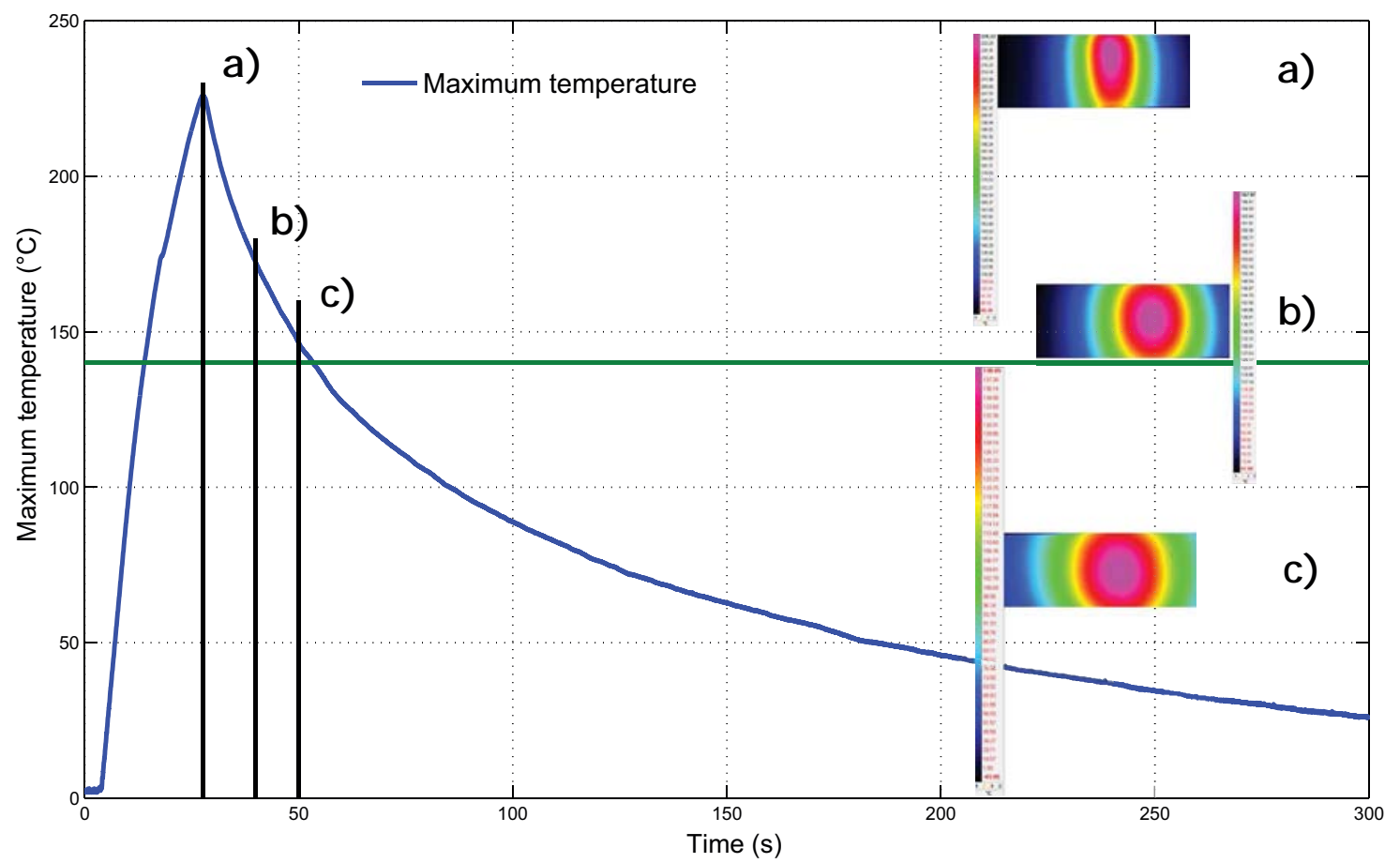

Fig. 13. IR thermogram during and after irradiation: temperature $\left({ }^{\circ} \mathrm{C}\right)$ versus time $(\mathrm{s})$. The vertical bold black lines indicate the time when the images were recorded.
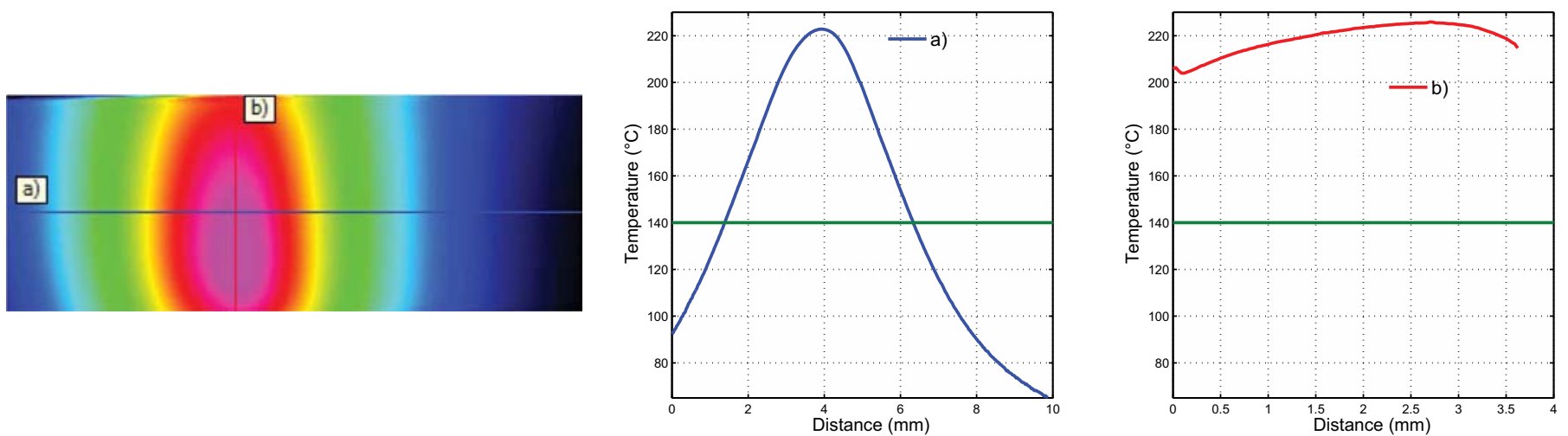

Fig. 14. IR profiles of the HAZ - a) Along $Y$ axis (lengthwise) - b) Along $Z$ axis (thickness).
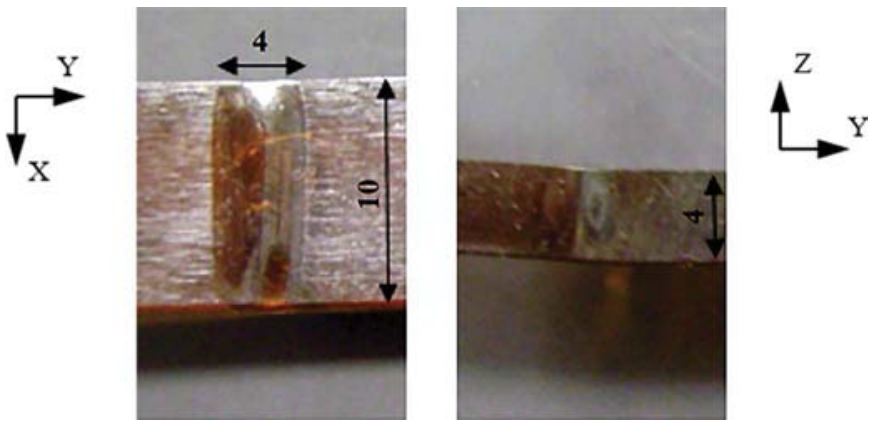

Fig. 15. Optical photography of the sample after laser beam/specimen interaction experiments: on the left: top view, on the right: side view.

Global reflection is the addition of the specular reflection and diffuse reflection. For the amorphous sample (A-PEKK), the global reflection factor is at most $12 \%$ at $1000 \mathrm{~nm}$.

From the transmission factor and the reflection factor, the absorbance factor $(\alpha)$ has been calculated. The results are shown in Fig. 10.
The absorbance is close to $100 \%$ for the lowest wavelength and decrease with wavelength. At $808 \mathrm{~nm}$, the absorbance is about $25 \%$.

To sum up, at $808 \mathrm{~nm}$, the transmission factor is about $60 \%$ for the quasi-amorphous sample, A-PEKK referred as transparent sample, whereas the transmission factor of the semi-crystalline C-PEKK is less than $3 \%$. The latter is considered as opaque material in the following. These results confirm what is supposed in other studies [21], the reflection factor is very low (less than $12 \%$ ) compared to transmission and absorption factor.

The black body is often used as a reference for determining the emissivity coefficient of materials. The black body absorbs light in the visible wavelength range and transmits in the infrared range. Despite polymer emissivity measurements have been shown to be highly imprecise $[20,21]$, polymers are usually considered to have high emissivity coefficients [12,22], their behavior within visible and IR range are close to those of the black body. Due to the novelty of the polymers of the PAEK family, a few optical properties are available in the literature. Nevertheless, their optical properties are expected to be the same as any high performance polymer in this wavelength range. To confirm this assumption, the transmission factor has been measured. The sensor used for IR thermography measurements covers a spectral range from 3.70 to 


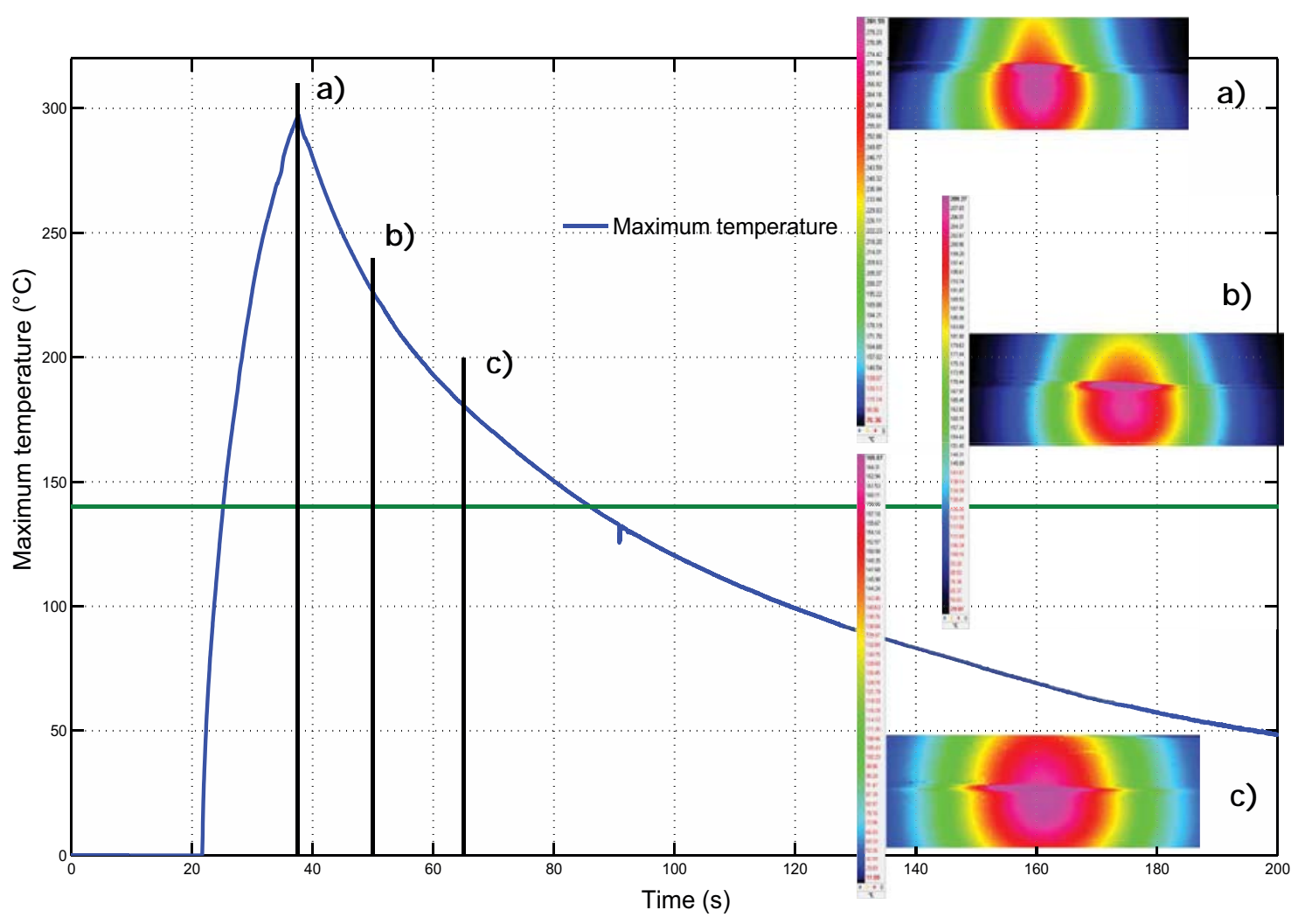

Fig. 16. IR thermogram for welding test: during and after irradiation of the assembly: temperature $\left({ }^{\circ} \mathrm{C}\right.$ ) versus time (s). The vertical bold black lines indicate the time when the images were recorded.

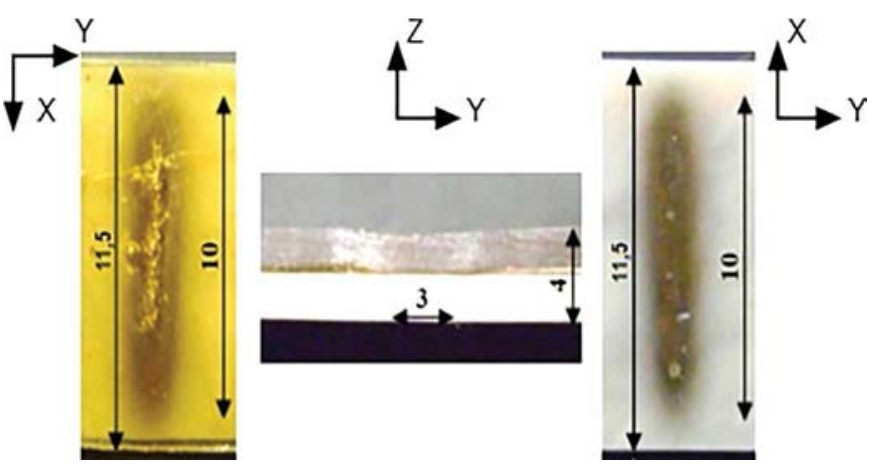

Fig. 17. Photography of the welded samples: On the left: top view of the assembly, on the middle: side view, on the right: bottom view of the assembly dimensions given in $\mathrm{mm}$.

$5.15 \mu \mathrm{m}$. In this range, the transmission is less than 5\% for A-PEKK and C-PEKK, as seen in Fig. 11.

Assuming like other authors [13] that the reflection factor is low, we conclude that the PEKK absorbs almost the whole incident radiation in the 3.70 to $5.15 \mu \mathrm{m}$ range.

The temperature calculated from the infrared thermography measurements depends on the emissivity of the materials. To estimate the impact of temperature variation due to the emissivity, a variability test was undertaken. The emissivity was set up as 0.91 for the semitransparent PEKK and 0.96 for the opaque one; these values are in agreement with previous studies $[12,23]$. For our variability test, the emissivity factor varies from 0.8 to 0.98 . The effect on the resulting maximum temperature is plotted in Fig. 12. The results are reported in Table 1, where $\varepsilon=0.91$ is chosen as a reference: variations of the maximum temperature are within the range of $-5.5^{\circ} \mathrm{C}-+9.9^{\circ} \mathrm{C}$. Standard deviation $\sigma$ and average $\mu$ of this data set are respectively $6.44{ }^{\circ} \mathrm{C}$ and $223.46^{\circ} \mathrm{C}$. The coefficient of variation $C_{v}$, can be calculated as follow:

$C_{v}=\left(1+\frac{1}{4 n}\right) \times \frac{\sigma}{\mu}$

where $n$ is the population, $\sigma$ is the standard deviation and $\mu$ is the average. With experimental data, $C_{v}$ is 0.03 . Due to the very low value of $C_{v}$, the following emissivity coefficients are finally used: 0.91 for the transparent material and 0.96 for opaque one.

\subsection{Laser beam/specimen interaction}

In order to set up all the infrared measurements, experiments were performed on a single sample. Laser power, measured in Watt, is set during a given time (s) upon the spotted area $\left(\mathrm{mm}^{2}\right)$ brings energy density in $\mathrm{J} . \mathrm{mm}^{-2}$. Energy density was $22 \mathrm{~J} . \mathrm{mm}^{-2}$ for the laser beam/specimen interaction tests. An IR movie was recorded using the parameters defined in the experimental section. All the images were processed seeking to match measured area to the specimen size. A plot of the maximum temperature and three images at different steps are provided in Fig. 13 to show a qualitative distribution of the thermal fields in the sample thickness.

Due to the integration time applied, only temperature above $140{ }^{\circ} \mathrm{C}$ is considered. This thermogram confirms that real irradiation time lasts about $20 \mathrm{~s}$. The heating rate is very fast; it is calculated at $600{ }^{\circ} \mathrm{C} \cdot \mathrm{min}^{-1}$ in average.

At the end of the irradiation at $22 \mathrm{~J}_{\mathrm{mm}} \mathrm{mm}^{-2}$, the maximum temperature reached is $222 \pm 7{ }^{\circ} \mathrm{C}$. This temperature is about $70^{\circ} \mathrm{C}$ higher than the glass transition of the polymer. The whole thickness $(2 \mathrm{~mm})$ of the sample is affected uniformly by the temperature rise. The heat-affected zone (HAZ) is wider in the direction parallel to the laser beam at the end of the irradiation. As soon as the irradiation is stopped, the HAZ becomes wider and wider due to heat conduction. After irradiation, thermal waves propagate perpendicularly to lead to a homogeneous circular 
shape HAZ in the observed plane, at the end of the experiment. The cooling rate of the sample is $270{ }^{\circ} \mathrm{C} \cdot \mathrm{min}^{-1}$ in the first $10 \mathrm{~s}$ and decreases to $150^{\circ} \mathrm{C} \cdot \mathrm{min}^{-1}$ in the following $20 \mathrm{~s}$. According to the results obtained by DSC, the temperature along the sample's thickness was higher than the glass transition $\left(150^{\circ} \mathrm{C}\right)$ during $34 \mathrm{~s}$ for an irradiation of $21 \mathrm{~s}$. The polymer does not reach its melting point with this time and power of exposure.

The dimensions of the HAZ is determined using profile on both directions for temperature above $150{ }^{\circ} \mathrm{C}$. In Fig. 14, both profiles bring HAZ size: $4 \mathrm{~mm}$ lengthwise and $4 \mathrm{~mm}$ all along the sample's thickness. The whole thickness is thermally affected, that matches with visual observation in Fig. 15: the HAZ appears on both surfaces of the sample, where the affected zones look brighter and smoother than the rest of the sample. The same is observed on the upper surface of the sample.

\subsection{Welding tests}

After the test of laser beam/specimen interaction, IR measurements were performed during welding of a couple of $2 \mathrm{~mm}$ thick specimens. A-PEKK was used as top element, C-PEKK for the bottom. The energy density was determined previously and fixed at $28 \mathrm{~J} . \mathrm{mm}^{-2}$ for this test.

The evolution of the maximum temperature of the assembly was recorded during welding, still with the camera sensor perpendicular to the lasersheet and perpendicular to the sample's length. The results are plotted in Fig. 16, as well as images at different steps of the process to have a qualitative distribution of the thermal fields.

As previously, only temperature above $140{ }^{\circ} \mathrm{C}$ is considered due to the integration time applied. Real irradiation time is about $16 \mathrm{~s}$. As expected in transmission laser welding, the energy of the beam is stopped at the interface since the lower part is absorbent at $808 \mathrm{~nm}$. The maximum temperature during the process is $295 \pm 7{ }^{\circ} \mathrm{C}$ for an irradiation at $28 \mathrm{~J} . \mathrm{mm}^{-2}$. This temperature is about $140{ }^{\circ} \mathrm{C}$ higher than the glass transition, and about $20^{\circ} \mathrm{C}$ higher than the beginning of the melting of PEKK occurring at $275^{\circ} \mathrm{C}$. The heating rate during irradiation is more than $600^{\circ} \mathrm{C} \cdot \mathrm{min}^{-1}$. Unlike for the laser beam/specimen interaction presented in Fig. 13, the heat-affected zone in the lower sample is symmetrical in both directions, alongside and perpendicularly to the lasersheet, whereas the HAZ is stretched out in the upper part.

When the irradiation is stopped, the temperature in the lower part gradually decreases. The cooling rate is about $300^{\circ} \mathrm{C} \cdot \mathrm{min}^{-1}$ the first $10 \mathrm{~s}$ just after the irradiation is stopped. The time needed to reach $\mathrm{T}_{\mathrm{g}}$ on cooling from the maximum temperature $\left(295^{\circ} \mathrm{C}\right)$ is $42 \mathrm{~s}$. The average cooling rate between the irradiation is stopped and the $\mathrm{T}_{\mathrm{g}}$ is about $200^{\circ} \mathrm{C} \cdot \mathrm{min}^{-1}$. From DSC results, we expect no crystallization of A-PEKK upon cooling, the cooling rate is too fast.

Upon cooling, the HAZ in the upper part increases, and more, the $\mathrm{HAZ}$ in the lower part tremendously stretches out. In all, the temperature at the interface of both samples was higher than the glass transition during $55 \mathrm{~s}$ and higher than the melting temperature during $5 \mathrm{~s}$ for an irradiation time of $16 \mathrm{~s}$.

The photography of the resulting assembly is presented in Fig. 17. The dimensions of the HAZ measured from these pictures are in agreement with the dimensions obtained by infrared images.

\section{Conclusion}

The temperature distribution on the surface along the sample's thickness has been measured during transmission laser welding. The originality of this work is to place the camera sensor perpendicular to the lasersheet and perpendicular to the welded interface. With such an experimental set up, the temperatures at the welded interface and along the thickness of the specimens have been measured. We are aware that the temperatures measured are not representative of the ones inside the sample ( $x$ axis), due to the difference of propagation of heat waves inside the material and in air. Nevertheless, the temperature measured on the surface of assemblies is helpful to link the process parameters (laser power and laser speed) to the mechanical strength of welded interfaces.

For this study, a high-performance thermoplastic, PEKK was chosen, its glass transition was measured at $150{ }^{\circ} \mathrm{C}$ and its melting range between 275 and $320^{\circ} \mathrm{C}$. The material was processed by compression molding to get quasi-amorphous samples, referred as A-PEKK and fully crystallized, referred as C-PEKK. Their optical properties as well as kinetics of crystallization have been outlined. It is the first time the laser welding of PEKK is reported.

The laser beam/specimen interaction test was performed with CPEKK irradiated at $22 \mathrm{~J} . \mathrm{mm}^{-2}$. The maximum temperature reached by the sample is about $220^{\circ} \mathrm{C}$ and the temperature along the sample's thickness stays above $\mathrm{T}_{\mathrm{g}}$ during $34 \mathrm{~s}$ when the irradiation step lasts $21 \mathrm{~s}$. When the irradiation is stopped, the heat-affected zone (HAZ) has initially a longitudinal shape along the whole thickness of the sample. The shape of the HAZ becomes symmetrical in the observed plane for $20 \mathrm{~s}$ after the irradiation is stopped.

For the welding test, an assembly made of a transparent (quasiamorphous) sample as the upper part and opaque (fully crystallized) sample as the lower part was used. The maximum temperature reached at the interface is about $295^{\circ} \mathrm{C}$ when the upper specimen is irradiated for $16 \mathrm{~s}$ with an energy density of $28 \mathrm{~J} . \mathrm{mm}^{-2}$. The temperature along the sample's thickness stays above $\mathrm{T}_{\mathrm{g}}$ during $55 \mathrm{~s}$ and above the melting temperature during $5 \mathrm{~s}$. This time is obviously enough to allow macromolecules to self-diffuse and to create entanglements. Indeed, the obtained assembly looks strong, although no mechanical test has been performed until now.

This grade of PEKK, with a slow kinetics of crystallization is suitable for laser transmission welding. With the energy beam and irradiation time studied, the maximum temperature inside the sample is kept far from the degradation of PEKK. Finally, the location and size of the heataffected zone (HAZ) have been determined. This work is a step towards more reliability of the laser welding process. In future works, the interfacial strength of welded assemblies will be studied by mechanical tests.

\section{Acknowledgment}

The authors would like to thank the French Ministry of Higher Education and Research for Financial support through a PhD funding.

\section{References}

[1] Tarrieu J. Etude et durabilité de solutions de packaging polymère d'un composant diamant pour l'électronique de puissance haute température $\mathrm{PhD}$ Thesis. Institut National Polytechnique de Toulouse; 2012.

[2] Wise RJ. Thermal welding of polymers. Abington Publishing; 1999. p. 21.

[3] Klein R. Laser welding of plastics. Wiley-VCH; 2012.

[4] Voyutskii SS. Autohesion and adhesion of high polymers. Wiley; 1963.

[5] Vasenin RM. Adhesion pressure in the diffusion theory of the adhesion of polymers. Polym Sci U.S.S.R 1962;3(4):608-15

[6] De Gennes PG. Comptes Rendus de l'Académie des Sciences 1980;B291:219-21 Paris(in French).

[7] De Gennes PG. Reptation of a polymer chain in a presence of fixed obstacles. J Chem Phys 1971;55(2):572-9.

[8] Wool RP, Yuan BL, McGarel OJ. Welding of polymer interfaces. Polym Eng Sci 1989;29(19):1340-67.

[9] Amanat N, et al. Transmission laser welding of amorphous and semi-crystalline polyetheretherketone for applications in the medical device industry. Mater Des 2010;31:4823-30.

[10] Potente $\mathrm{H}$, et al. Investigations towards application of a new technique on laser transmission welding. Weld World 2001;45(5-6):15-20.

[11] Ilie M, et al. Through-transmission laser welding of polymers - field modeling and infrared investigation. Infrared Phys Technol 2007;51:73-9.

[12] Speka M, et al. The infrared thermography control of the laser welding of amorphous polymers. NDT\&E Int 2008;41:178-83.

[13] Akué Asséko A, et al. Laser transmission welding of composites-part A: thermo-physical and optical characterization of materials. Inf Phys Techol 2015;72:293-9.

[14] Akué Asséko A, et al. Laser transmission welding of composites-part B: experimental validation of numerical model. Inf Phys Techol 2015;73:304-11.

[15] Gouesbet G, Gréhan G. Generalized Lorenz-Mie theories, from past to future. Atomic Sprays 2000;10(3-5):277-334.

[16] Gouesbet G, Gréhan G. Generalized Lorenz-Mie theories for assemblies of spheres and aggregates. J Opt 1999;1:706-12. 
[17] Blanca CM, Saloma C. Monte Carlo analysis of two-photon fluorescence imaging through a scattering medium. Appl Opt 1998;37(34):8092-102.

[18] Jonas A, Legras R, Issi JP. Differential scanning calorimetry and infrared crystallinity determinations of poly(aryletheretherketone). Polymer 1991;32(18):3364-70.

[19] Mehmet-Alkan AA, Hay JN. The crystallinity of poly(ether ether ketone). Polymer 1992;33(16):3527-30.

[20] Becker F. A step towards understanding the heating phase of laser transmission welding in polymers. Polym Eng Sci 2002;42(2):365-74.
[21] Van de Ven J, Erdman A. Laser transmission welding of thermoplastics - part II: experimental model validation. Manuf Sci Eng 2007;129:859-67.

[22] Ilie M, et al Laser beam scattering effects in non-absorbent inhomogenous polymers. Opt Lasers Eng 2007;45:405-12.

[23] Cosson B, et al. Infrared heating stage simulation of semi-transparent media (PET) using ray tracing method. Int J Mater Form 2011;4(1):1-10. 

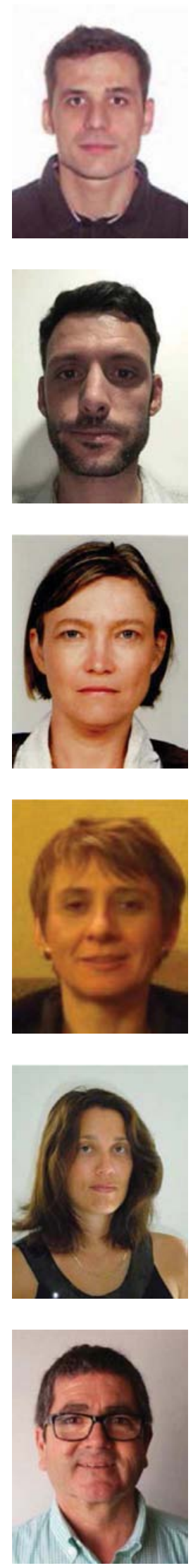

M. Villar is a mechanical engineer with a specialization in materials science, thermal and energy technologies. After being involved in some industrial projects, he went on to develop his scientific profile with a PhD thesis in Polymer Physics. His PhD thesis topics apply the transmission laser welding to assemble a new kind of polymer of the PAEK family. For the time being, the works he has accomplished covers the thermal, optical and mechanical characterization of materials and processes.

C. Garnier has been graduated by the National Engineering School of Tarbes. In 2011, he defended his PhD thesis untitled "Dynamic Behavior of LRI's composite structures: application to impact and fatigue". In 2013, he joined the Laboratory of Manufacturing Engineering in Tarbes, part of the University of Toulouse, France, as associate professor. His research interests include all aspects of composite materials (manufacturing, behavior, FEM, inspection, tests). His research specially focuses on composite material behavior in static, fatigue and multiaxial fatigue, on experimental mechanics and on thermal fields measurements.

F. Chabert obtained a PhD degree in Materials and Process Engineering in 2004 at the Institut National Polytechnique of Grenoble - France in the field of materials for fuel cells. She worked as postdoctoral fellow successively at the Chemical Engineering Department at the University of Melbourne, Australia and ESPCI Paristech, France. Since 2010, she is a teacher-researcher at the Interface and Functional Materials group of the Laboratory of Manufacturing Engineering in Tarbes, part of the University of Toulouse. Her research focuses on polymer processing, assembling processes for thermoplastics as well as nano and micro-composites with controlled properties. She has co-authored 4 patents and 15 scientific articles.

from the is Professor of materials engineering at the National Engineering School of Tarbes (ENIT) since 2012. She graduated with a Ph.D. in Physics physical, chemical and mechanical properties of adhesive interfaces in multi-materials and on interface durability. The main aim is the establishment of reliable relationships between the polymeric (adhesive or resin matrix) structure and its properties such as mechanical, rheological, thermal and adhesives into the interface.

D. Samélor has been graduated in Materials Engineering by Evry-Val-d'Essonne University in 2001. She completed her Masters with specialization on methods of preparation and characterization of thin films in the same university in 2002. In 2003, she joined the CIRIMAT laboratory in Toulouse as an engineer. Her activities are focused on preparation of ceramics, metals and composites by Metal Organic Chemical Vapor Deposition (MOCVD) and characterization of the coatings by conventional or more specific techniques.

J. C. Diez teaches at the University of Zaragoza's School of Engineering and Architecture (Department of Materials Science). He holds a Physics Degree and Ph.D. from the University of Zaragoza. Since 1989 he teaches in this University, in subjects mainly related with General Physics, Materials Science, Technology of Ceramics and Materials Laser Processes. He is mainly interested on the use of laser technology to improve the properties of functional ceramics (HTc superconductors and thermoelectric oxides). He has also experience in the use of laser radiation on metals, polymers, and composite materials, especially on the mold sector and in aeronautic applications. 


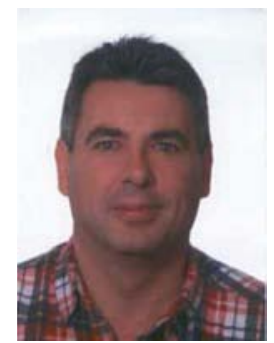

A. Sotelo had his Doctoral degree in Chemistry at the Universidad de Zaragoza in 1994. He has got a two-years postdoctoral position at Max-Planck Institute for Metals Research in Stuttgart (Germany) in 1995. In 1997 he got two years Marie Curie grant in CRISMAT Laboratoire, Caen (France). From 2000 he is Professor at the Universidad de Zaragoza in the Department of Materials Science. He has published more than 135 scientific articles and book chapters, most of them related to thermoelectric and superconducting ceramics with emphasis on laser processing techniques.

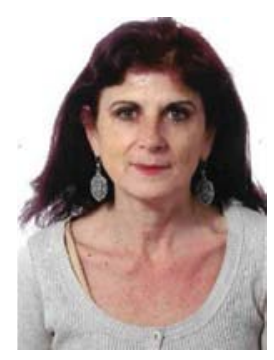

M. A. Madre has been graduated in Chemistry by the University of Zaragoza. She has got Ph.D. in Engineering by the Universidad de Zaragoza with qualification: Cum Laude with Extraordinary Doctorate Award. Since 1991 she is University Professor at the Dpt. Materials Science in the School of Engineering and Architecture. Her research interests include aspects of: Thermoelectric and superconducting ceramics. Texturing of ceramics by laser techniques, laser appliances in other fields, solution chemical synthesis techniques, and mechanical properties. She has published more than 110 scientific articles and book chapters. 